\title{
Marcel RIOUX (1919-1992)
}

sociologue, Université de Montréal

(1964)

\section{"Le socialisme aux Etats-Unis"}

Un document produit en version numérique par Jean-Marie Tremblay, bénévole, professeur de sociologie au Cégep de Chicoutimi

Courriel: jimt sociologue@,videotron.ca

Site web pédagogique : http://www.uqac.ca/jmt-sociologue/

Dans le cadre de la collection: "Les classiques des sciences sociales"

Site web: http://www.uqac.ca/Classiques_des_sciences_sociales

Une collection développée en collaboration avec la Bibliothèque

Paul-Émile-Boulet de l'Université du Québec à Chicoutimi

Site web: http://bibliotheque.uqac.uquebec.ca/index.htm 
Cette édition électronique a été réalisée par Jean-Marie Tremblay, bénévole, professeur de sociologie au Cégep de Chicoutimi à partir de :

Marcel Rioux, "Le socialisme aux États-Unis". Un article publié dans la revue Socialisme 64, Revue du socialisme international et québécois, no 1, printemps 1964, pp. 87-107.

M. Marcel Rioux (1919 - 1992), sociologue, enseignai la sociologie à l'Université de Montréal.

Polices de caractères utilisée :

Pour le texte: Times, 12 points.

Pour les citations : Times 10 points.

Pour les notes de bas de page : Times, 10 points.

Édition électronique réalisée avec le traitement de textes Microsoft Word 2001 pour Macintosh.

Mise en page sur papier format

LETTRE (US letter), 8.5' x 11'”)

Édition numérique réalisée le 6 novembre 2004 à Chicoutimi, Ville de Saguenay, province de Québec, Canada.

\section{Fait avec}

Macintosh 


\title{
Table des matières
}

\author{
Introduction
}
A. Qu'est-ce que le socialisme ?
B. Socialisme et industrialisation
C. Les socialismes aux États-Unis
D. Socialisme utopique aux U.S.A.
D. Caractères généraux de la société américaine
F. Classe ouvrière et mobilité sociale
G. Le système politique américain.
H. Idéologie américaine: Idéologie-substitut
I. Morale et politique

En guise de conclusion 


\title{
Introduction
}

\begin{abstract}
"Socialisme 64" n'entend pas éluder les questions les plus difficiles; c'est au contraire celles qui l'intéressent le plus. Commençons par celles-ci : "Il n'y a jamais eu de socialisme aux U.S.A., Pourquoi y en aurait-il au Québec ? Mais quel est le cheminement du Socialisme aux U.S.A.? Cet article veut rouvrir ce dossier. Quant à l'avenir du socialisme au Québec, "Socialisme 64" est un chantier qui s'ouvre pour répondre précisément à cette question.
\end{abstract}

$\underline{\text { Retour à la table des matières }}$

Au début du siècle, en 1900 très précisément, l'économiste allemand, Werner Sombart, écrivait un petit volume intitulé :

“Pourquoi n'y a-t-il pas de socialisme aux États-Unis ?” Plusieurs auteurs depuis Sombart ont tenté d'expliquer ce phénomène. Nous nous posons à notre tour la même question, non pas tant pour apporter de nouvelles réponses que pour prendre la mesure du défi que pose la création d'un mouvement de pensée et d'action socialistes au Québec. Avant de nous demander si le socialisme a de l'avenir aux États-Unis, et de démontrer que le Québec est bien différent du pays capitaliste le plus avancé et que ces deux pays peuvent évoluer bien différemment, il faut nous demander pourquoi le socialisme a connu jusqu'à aujourd'hui si peu de succès électoraux aux États-Unis. C'est cette question qui fait l'objet du présent article. 


\section{A. Qu'est-ce que le socialisme?}

$\underline{\text { Retour à la table des matières }}$

Et tout d'abord que faut-il entendre par socialisme ? On dit souvent qu'il existe autant de variétés de socialismes qu'il naît de groupuscules de gauche dans tel ou tel pays. Il existe une grande part de vérité dans ces assertions. Parce que le socialisme est, dans sa visée profonde, démocratique et idéologique, il n'est pas surprenant que des groupes d'individus diffèrent sur leurs façons d'envisager ce que doit être la bonne société et formulent des opinions qui diffèrent entre elles. Ces divergences mises à part, il existe quand même un fond commun à tous les socialismes et qu'on peut rattacher à deux grands phénomènes du monde moderne: l'idéologie démocratique et l'industrialisation des sociétés occidentales. Ces deux Phénomènes sont en réalité étroitement liés: la Révolution française, où s'est d'abord exprimée cette idéologie démocratique, trouve elle-même ses causes profondes dans les bouleversements économiques et sociaux du XVIIIe siècle. Si toutefois, on envisage l'idéologie démocratique en elle-même, on se rend compte qu'elle renferme deux idées principales : la liberté et l'égalité des personnes humaines. Les deux idées sont intimement liées, parce que la liberté que réclame la démocratie, c'est la liberté pour tous et non seulement pour quelques-uns; ce qui implique l'établissement d'une certaine forme d'égalité entre les hommes. Si ces deux idées, liberté et égalité, sont liées, elles ne sont pas inséparables. Dans certains pays, à certaines périodes, c'est l'idée de liberté qui prédomine; c'est ainsi qu'en Angleterre, aux XVIlle et XIXe siècles, on verra se développer dans ce pays, encore largement aristocratique, des formes de libertés individuelles encore ignorées ailleurs. Napoléon, au contraire, continuant la poussée de la Révolution française qui proclamait les deux - liberté et égalité a eu tendance à maintenir l'égalité au détriment de la liberté. On peut aussi dire, en gros, que l'aspiration à la liberté a été l'affaire des individus et que l'aspiration à l'égalité, celle des classes dominées, parce que le socialisme et les partis qui s'en réclament ont voulu être le parti des travailleurs, l'aspiration à l'égalité y a été mise de l'avant plus que dans les autres partis de gauche, le radicalisme surtout. Ce fut l'intention des partis radicaux-socialistes d'unir ces deux aspirations. En France nommément, le parti radical-socialiste est vite retombé sur sa lancée radicale. On peut donc dire que le projet fondamental du socialisme fut et demeure l'extension de la démocratie - liberté et égalité - au régime économique et social; il vise à faire disparaître cette antinomie qui 
surgit entre la démocratie politique et le régime économique du capitalisme. Comment espérer réaliser la démocratie politique, comment vouloir donner à tous les hommes les mêmes droits dans l'ordre politique et leur refuser ces droits dans le domaine économique ? Selon les conditions économiques particulières à chaque pays, il appartient à chaque parti socialiste - et ce sera notre tâche au Québec - d'étudier comment, concrètement, on peut réaliser l'extension de la démocratie politique au régime économique. En somme, c'est la base commune de tous les partis socialistes; seuls diffèrent les modalités et les moyens d'action.

\section{B. Socialisme et industrialisation}

$\underline{\text { Retour à la table des matières }}$

Le mot socialisme est d'usage récent: il naît entre 1820 et 1840, en France et en Angleterre, avec les mouvements qui s'opposent aux conséquences économiques et sociales de l'industrialisation de l'Europe. On distingue communément deux grandes espèces de socialisme : utopique et scientifique. En gros, le socialisme utopique, qu'il soit religieux ou profane, a tendance devant les conditions misérables des masses au XIXe siècle, à préconiser des remèdes et des types d'organisation sociale qui empruntent beaucoup au passé. Le socialisme scientifique, au contraire, se fondant sur l'analyse de la société industrielle, veut essayer de la dépasser et construire une société de l'avenir plutôt que de retourner au passé. Disons que les deux socialismes sont d'accord sur la critique de la société née du capitalisme concurrentiel mais qu'ils diffèrent sur les remèdes et les correctifs qu'il faut apporter. Owen et Fourier, ayant d'abord employé le mot socialisme pour désigner leurs systèmes, Marx, qui les qualifie d'utopistes, emploie lui-même le mot communisme pour s'en différencier; socialisme dénote, de plus, une action réformiste et graduelle tandis que communisme désigne une action radicale et révolutionnaire.

On peut classer les différents socialismes selon la nature de leurs buts: les socialismes religieux croient que seule une réforme spirituelle peut régénérer l'humanité et corriger les abus de la société industrielle et du capitalisme. Les socialismes profanes, marxistes ou non, tiennent que si l'on veut vraiment changer la vie, il faut réorganiser complètement la société et mettre sur pied d'autres relations économiques entre les individus. Les individualistes et les 
anarchistes, Proudhon et Kropotkine, entre autres, veulent associer davantage les individus à cette réforme et prônent une action volontariste des individus.

Chacun de ces grands types de socialisme privilégie un type d'organisation pour lutter contre le capitalisme et hâter le passage à la société de demain; pour les socialistes utopiques, c'est la petite communauté homogène d'un autre âge; pour les ouvriers organisés, ce sont les coopératives et les syndicats; pour les sociaux-démocrates du type "fabian", c'est l'État; pour les marxistes, c'est le Parti. Chacun se situe aussi dans le temps : les socialistes utopiques veulent retourner dans le passé; les socialistes bourgeois veulent accorder aux ouvriers des mesures de bien-être si nombreuses et si importantes qu'ils oublieront de combattre la structure économique et sociale des États capitalistes; c'est le présent qu'ils privilégient. Enfin, les socialistes marxistes, communistes ou autres, sont nettement orientés vers l'avenir; c'est une société nouvelle qu'ils veulent bâtir; ils ne veulent pas retourner au passé, ni s'accommoder du présent, mais bâtir une société neuve.

\section{Les socialismes aux États-Unis}

$\underline{\text { Retour à la table des matières }}$

Quand Sombart veut savoir pourquoi il n'y a pas de socialisme aux ÉtatsUnis, il ne veut pas dire qu'il n'y a aucune organisation ni aucun mouvement socialiste dans ce pays; il ne parle que de la faiblesse électorale du parti socialiste aux U.S.A. Au début du XXe siècle, comme aujourd'hui, les U.S.A. se distinguaient par l'absence de députés socialistes. La plupart des pays européens, au contraire, comptaient un nombre imposant d'élus socialistes juste avant la guerre de 1914; l'Autriche-Hongrie en avait élu 82, la Belgique 39, Le Danemark 32; l'Allemagne 119, la France 103, l'Angleterre 42, la Hollande 16; l'Italie, 80; la Finlande, 90; la Norvège, 23; la Russie, 24 et la Suède 73. Depuis ce temps, les partis socialistes de tous ces pays ont connu des fortunes diverses; au Danemark, le parti socialiste est au pouvoir depuis les années 30; en France et en Angleterre, par exemple, des partis socialistes ont détenu le pouvoir, seuls ou avec d'autres groupements politiques. Depuis la décolonisation de l'Afrique et de l'Asie, des partis socialistes, plus ou moins puissants, sont apparus dans la plupart des pays de ces continents ainsi qu'en Amérique du Sud. Seuls les U.S.A. continuent, comme au temps de Sombart, à faire exception et à se présenter comme le dernier rempart du capitalisme. 
Pourquoi? Si le socialisme n'a pas une forte clientèle électorale aux ÉtatsUnis, ce n'est pas faute d'organisations socialistes; tous les types de socialismes, depuis les utopismes religieux jusqu'à trotskysme, ont pénétré dans ce pays et ont été proposés au public. L'apparition des différents socialismes aux U.S.A. suit à peu près la même chronologie que celle des variétés européennes. En Europe comme aux U.S.A., le socialisme suit la même évolution que la société elle-même: au XVIle siècle, les utopies pré-socialistes s'incarnent dans une Europe encore largement religieuse et sont elles-mêmes imprégnées de religion; le rationalisme du XVIIIe siècle rend les mouvements de réforme plus profanes; ce n'est qu'au XIXe siècle qu'apparaît le socialisme dit scientifique.

Ce serait trop simplifier que de dire que seul le socialisme religieux a été influencé par le christianisme. La visée essentielle du socialisme, utopique et scientifique, celle de l'égalité de tous les hommes, de la perfectibilité de l'homme, de la justice pour tous, se retrouve dans le christianisme. Et il semble bien que dans les pays anglo-saxons la liaison entre le christianisme et le socialisme ait été particulièrement forte; c'est cette caractéristique qui rend compte semble-t-il, du pullulement des organisations et des idées socialistes aux U.S.A. mais aussi de leur faiblesse politique.

\section{Socialisme utopique aux U.S.A.}

\section{Retour à la table des matières}

Le socialisme religieux ou sectaire connut un certain succès aux U.S.A., particulièrement à partir de la décennie 1734-1744, époque de la Grande Renaissance évangélique; plus tard les Shakers qui forment des groupes où la propriété, la production et la consommation sont mises en commun, se développeront à la fin du XVIIIle siècle et pendant tout le XIXe siècle. Engels, en 1845, donnait en exemple les communautés Shakers qui, selon lui, avaient réalisé certains objectifs du socialisme. "Dans leurs dix villages, il n'y a pas un seul policier, pas de juge, pas d'avocat, pas de soldat, pas de prison ni de pénitencier; et pourtant tout marche régulièrement. Les lois du pays n'existent pas pour eux et elles auraient pu tout aussi bien être abolies sans que personne y fasse attention, car ils sont les gens les plus pacifiques et n'ont jamais livré 
un délinquant à la prison. Ils vivent comme on l'a dit dans la plus complète communauté de biens, et ni commerce ni argent n'ont cours parmi eux." 1 D'autres sectes, les Rappites, les Séparatistes Zoar et les perfectionnistes Oneida se développèrent pendant tout le XIXe siècle. Ces premiers socialistes étaient des illuminés religieux qui formèrent des petites communautés rurales dans lesquelles les ressources étaient mises en commun; ces communautés étaient homogènes, statiques et fermées. Plusieurs sectes protestantes et plus particulièrement le calvinisme ont toujours voulu essayer de concilier l'idéal chrétien et l'idéal social du socialisme. Selon Albert Mollengen ${ }^{2}$, qui a étudié le fondement religieux du socialisme occidental, si le socialisme chrétien a quelque chance de réussir aux U.S.A., ce seront des théologiens néo-calvinistes comme Reinhold Niebuhr, Paul Tillich et Edward Heimann qui seront au centre de ce mouvement.

Les partisans américains d'un socialisme utopique profane, avaient, au contraire, des visées terrestres; leurs mouvements se situaient sur la terre et dans l'histoire; ils ne se fiaient pas surtout à la mystique ni à la religion pour atteindre leurs buts. Ces socialistes, contrairement aux Shakers, réagissaient directement contre les abus de la société industrielle naissante en proposant divers degrés de propriété collective pour fournir aux individus les moyens de se réaliser pleinement. Encore ici, les socialistes diffusèrent et essayèrent d'appliquer des idées venues d'Europe, plus particulièrement de France et d'Angleterre. Owen, Fourier et Cabet furent les principaux initiateurs de ces mouvements américains.

C'est aux U.S.A. qu'Owen, le fondateur du mouvement coopératif en Angleterre, décida de fonder ses colonies socialistes. En 1824, il vint en Amérique et fonda des villages d'amitié et de coopération à New-Harmony dans l'Indiana et au Texas. Les caractères principaux de ces communautés, comme l'abolition de la propriété individuelle, se rapprochent de ceux des communautés sectaires. Mais ils en diffèrent aussi par la tentative d'abolir la religion et les liens légaux en matière sexuelle. Mais encore ici, la différence principale entre ces communautés et phalanstères américains et ceux d'Europe, c'est qu'ils restent beaucoup plus religieux en Amérique qu'en Europe; le sentiment religieux est lié davantage au socialisme aux U.S.A. qu'en Europe.

Dans ce court article, ce ne sont pas ces variétés de socialisme qui nous intéressent; pas plus en Amérique qu'ailleurs dans le monde, le socialisme utopique, religieux ou profane, n'a eu de suite politique mais il a servi de précurseur au socialisme dit scientifique. C'est cette dernière variété qui devait

Cité par Henri Desroches : "Les Shakers américains", p. 289.

Mollegen, Albert T.: "The Religious Basis of Western Socialism" dans "Socialism and Arnerican Life" (Egbert et Parsons) pp. 99-123. 
servir de base à l'action politique, aux partis politiques et à la prise du pouvoir dans différents pays.

Pourquoi, en somme, les différentes variétés de socialisme utopique ontelles eu autant et sinon plus de succès aux U.S.A. qu'en Europe alors que le socialisme dit scientifique n'a pas eu de succès comparables à ceux qu'il devait connaître en Europe et dans le reste du monde ? Si le socialisme peut être envisagé comme le prolongement dans le domaine économique de la démocratie politique, on peut se demander pourquoi les Américains, si férus de démocratie, ont toujours fait grise mine à ce mouvement ? Les raisons que les différents auteurs qui ont étudié cette question ont mises de l'avant peuvent être classifiées en cinq larges catégories : 1 . le libéralisme; 2 . la force ouvrière et la mobilité sociale; 3 . la politique américaine; 4. l'idéologie américaine; 5. la morale et la politique.

\section{E. Caractères généraux de la société américaine}

$\underline{\text { Retour à la table des matières }}$

Au moment où dans la plupart des pays européens naissaient le socialisme et les partis politiques socialistes, les États-Unis d'Amérique étaient encore en plein développement et leurs frontières restaient ouvertes. Comparée aux pays européens, l'Amérique se présentait comme un continent neuf aux possibilités illimitées. Ce qui fait dire au sociologue américain Lipset que les U.S.A. sont en vérité la première "nouvelle nation" qui devait naître de la décolonisation. Contrairement aux pays européens qui avaient atteint leurs limites géographiques depuis longtemps, les États-Unis avaient devant eux un continent entier à découvrir, à coloniser et à exploiter. Ceux qui n'étaient pas satisfaits de l'ordre des choses pouvaient partir pour l'ouest du pays et aller se bâtir là une vie à leur gré. Il est bien évident aussi que la mobilité sociale était extrêmement grande au XIXe siècle; le vieux mythe américain que n'importe qui peut devenir président des États-Unis ou de General Motors a pris naissance à cette époque, alors que de telles possibilités existaient vraiment. Les Américains sont les descendants des Anglais qui se sont battus pour les libertés individuelles; en Amérique, ces fils d'Anglais se sont ensuite battus pour leur liberté nationale qu'ils ont obtenue à la suite de la guerre de l'Indépendance. Comme la mobilité horizontale et verticale existait pour plusieurs couches de 
la population, les Américains n'étaient pas enclins à s'intéresser à l'égalité économique de tous les individus puisque cette inégalité bénéficiait à une bonne proportion de la population.

De plus, le seul modèle économique qu'ils connaissaient pour assurer la croissance et le développement économiques de leur pays était le modèle du capitalisme concurrentiel qui requiert une très grande mobilité sociale et beaucoup d'inégalité entre les individus. S'étant battus pour la démocratie politique, pour les libertés individuelles, ils n'étaient enclins à limiter la liberté économique de certains individus pourvu que leur société crût et se développât aussi rapidement qu'il était possible. Comme le standard de vie des Américains s'accroissait sans cesse, le mécontentement de certaines couches économiquement faibles était sporadique et limité à certains secteurs temporairement mal en point. Sombart a pu écrire qu'aux U.S.A. le socialisme s'est brisé sur les récifs du rosbif et de la tarte aux pommes. Pour les Américains, démocratie et capitalisme de concurrence sont apparus comme les deux formes, l'une politique, l'autre économique, d'une même doctrine: le libéralisme.

\section{F. Classe ouvrière et mobilité sociale}

$\underline{\text { Retour à la table des matières }}$

Dans tous les pays où le socialisme a réussi à s'implanter, c'est à la classe ouvrière qu'il le doit; c'est sur elle que les partis politiques se sont traditionnellement appuyés dans leur lutte contre le capitalisme pour instaurer la démocratie économique. Or, si cette classe ouvrière existait nombreuse et puissante aux États-Unis, il lui a toujours manqué aux moments névralgiques de son histoire une conscience de classe suffisamment développée pour appuyer les formations politiques socialistes. Pourquoi ? Encore une fois, on doit se rabattre sur la situation particulière des États-Unis. A cause de l'immigration constante et importante qui a déferlé sur ce pays pendant toute son histoire et particulièrement au XIXe siècle, il n'a jamais existé de classe ouvrière fixe et stable qui, comme dans les autres pays d'Europe, s'est développée sur place et a acquis une relative autonomie. Chaque génération d'ouvriers immigrants prenait place au bas de l'échelle sociale des U.S.A., déplaçait vers le haut ceux qu'elle remplaçait et espérait, elle-même, céder sa place à la nouvelle vague qui arrivait bientôt d'Europe. Ceux-ci, imbus de socialisme et d'une conscience de classe bien vivante, les perdaient bien vite, 
devant la fragmentation de la classe ouvrière américaine et devant les chances d'avancement que présentait le capitalisme concurrentiel.

De plus, ces vagues successives d'immigrants rendaient la classe ouvrière américaine extrêmement hétérogène; une grande partie de cette classe ouvrière, de ces ouvriers manuels, provenait de stocks ethniques différents : irlandais, italiens, polonais, allemands, entre autres, qui parlaient des langues différentes, pratiquaient des religions différentes et possédaient des traditions nationales bien caractérisées. Or, une classe sociale n'est pas seulement un assemblage d'individus qui pratiquent les mêmes métiers et gagnent à peu près le même salaire; l'ensemble de ces individus dans les pays où la mobilité sociale et ethnique n'est pas aussi grande qu'aux U.S.A., forment une sousculture bien caractérisée; c'est-à-dire que la classe sociale est aussi un style de vie caractérisé par des habitudes linguistiques, alimentaires, vestimentaires, par un système de valeurs commun et par une vision du monde commune; aux États-Unis, à cause de la trop grande hétérogénéité culturelle des ouvriers, cette sous-culture qu'est aussi la classe sociale n'a jamais réussi à se former. Un organisateur syndical écrivait dans les années 1920 cette lettre au professeur Sidney Hook : "J'ai été en rapports assez étroits avec les syndicats. je sais, par exemple que dans la ville de Havenshill (Mass.) où, pendant quelque temps, j'essayai d'organiser un syndicat, les facteurs décisifs parmi les masses travailleuses : sexe, religion, nationalité, etc., sont tellement puissants que bien que tous les ouvriers soient cordonniers, ils n'ont pas un trait en commun. Ils agiront plutôt en tant qu'hommes et femmes (les femmes reçoivent plus de travail que les hommes) en tant que: Irlandais, Grecs, Italiens et que catholiques, protestants ou juifs, qu'en tant que classe ouvrière." 3

Mais la grande mobilité sociale dans cette société ouverte et mouvante qu'étaient les U.S.A. au XIXe siècle a pris fin au début du XXe siècle, environ l'époque de la première guerre mondiale.

Depuis cette période, les classes sociales et les groupes socio-économiques ont eu tendance à apparaître avec plus de netteté. La mobilité sociale a elle aussi changé de nature; les individus entrent de moins en moins dans la société à titre de marchands ou d'industriels, mais comme employés d'organisations bureaucratiques. Le succès des individus n'est plus jugé par les seules forces aveugles du marché, comme c'était le cas de l'époque du capitalisme concurrentiel, mais par les supérieurs des organisations bureaucratiques. De plus, l'instruction devenant de plus en plus un pré-requis, un élément de mérite, un élément rationnel s'insère de plus en plus dans le système économique. Ces deux éléments - appareil bureaucratique et instruction - n'ont certes pas éliminé l'inégalité sociale, à cause du caractère oligarchique des

3 Hook, Sidney, "Pour comprendre Marx", Paris, 1936, p. 187. 
grandes corporations et du coût élevé de l'instruction, mais ils ont changé la nature de la mobilité sociale.

Plusieurs observateurs de la vie américaine ont fait remarquer qu'à cause des conditions particulières du développement économique des U.S.A., le prolétariat américain a très tôt perdu sa combativité et que, sous Gompers spécialement, la seule ambition du travail organisé fut de devenir une institution aussi légitime et aussi respectable que le monde des affaires et de l'Église. (Rappelons ici que Gompers, à titre d'organisateur et de président de l'American Federation of Labor, dont la fondation remonte à 1886. a joué un rôle considérable dans le mouvement ouvrier américain; il a laissé son nom au syndicalisme pragmatique et d'affaires qui, tout en n'appuyant aucun parti, essaie de tirer le maximum d'avantages pour les syndiqués de tous les partis politiques en présence.) Les syndicats américains en sont venus à se contenter de vouloir partager le gâteau de la prospérité américaine sans chercher à renverser le système capitaliste. Pourquoi le syndicalisme américain en est-il venu à prendre cette position ? Si on se rappelle que Gompers était lui-même marxiste au moment de la fondation de l'A.F.L. et, comme le dit Dwight MacDonald, "le principal grief qu'ils [les syndiqués de l'A.F.L.] opposaient aux Chevaliers du Travail était le caractère de classe "amorphe" et le manque de programme spécifiquement prolétarien," on constate qu'il a changé de politique en s'américanisant. En 1875, Gompers écrivait, en vue de la fondation de l'A.F.L., la lettre suivante aux ouvriers : "Sur tout le territoire des U.S.A. existent de nombreux corps organisés de travailleurs qui proclament que la dépendance avilissante du travailleur par rapport au capitalisme, maître de ses moyens d'existence, est la cause majeure de l'état de dégradation intellectuelle, morale et économique qui afflige la société et que tout mouvement politique doit être subordonné au premier grand objectif social qui est l'émancipation économique de la classe ouvrière." Et le préambule à l'acte constitutif adopté dix ans plus tard par l'A.F.L., acte qui est toujours à son programme officiel, commence par un écho de ce coup de tonnerre que fut le Manifeste communiste... "un combat met aux prises, dans toutes les nations du monde civilisé, les oppresseurs et les opprimés ... les capitalistes et les travailleurs." ${ }^{4}$ On peut donc reposer la question : pourquoi le socialisme a-t-il disparu des U.S.A. depuis la première grande guerre? Comme l'écrit Dwight MacDonald: "Entre la guerre de Sécession et la première guerre mondiale, il se produit en Amérique plusieurs mouvements de masse fondés sur la perspective d'une transformation fondamentale de la société: Chevaliers du Travail, I.W.W., parti socialiste de Debs. En 1910, par exemple, le parti socialiste comptait 58,000 cotisants et éditait 29 hebdomadaires de langue anglaise, 22 en langues étrangères, 3 quotidiens en anglais et 6 en langues étrangères. Vers 1912, le parti avait 126,000 membres; Debs recueillit près d'un million de suffrages aux élections présidentielles; de puissants syndicats

4 Cité par Dwight MacDonald : "Partir de l'homme", p. 45 et suivantes, Paris, 1948. 
comme les United Mine Workers étaient en majorité socialistes, et cette année-là, au congrès de l'A.F.L., le candidat socialiste à la présidence, luttant contre Gompers, recueillit plus du tiers des voix. Aux dernières élections à la présidence des U.S.A. (1948) le candidat socialiste recueillit moins de voix qu'il y avait de membres inscrits au parti en 1910. L'éclipse des Wobblies (I.W.W.) est plus complète encore: avant 1914, ils constituaient l'une des forces principales du prolétariat américain, dirigeaient des grèves impliquant des centaines de milliers d'ouvriers, préconisaient et appliquaient une doctrine de lutte des classes à outrance, basée sur une philosophie libertaire pratiquement anarchiste. Le radicalisme américain fit de grands progrès jusqu'en 1914." La guerre fut le roc sur lequel il se brisa. La raison de ce déclin, de cet échec, il ne faut peut-être pas la rechercher dans le mouvement ouvrier luimême, mais plutôt dans la vie politique américaine et dans son système politique lui-même.

\section{G. Le système politique américain.}

\section{Retour à la table des matières}

Plusieurs auteurs ont cherché à expliquer ce changement dans les buts du travail organisé et la faillite du socialisme américain après $1914,{ }^{5}$ en examinant de plus près la nature de la société américaine, et de son système politique. Deux types d'explication ont été donnés : le caractère agraire du socialisme américain et le fédéralisme des U.S.A. Le premier type d'explication peut éclairer la situation politique avant 1914 et ne retiendra pas longtemps notre attention. On a souvent fait remarquer que le fondement agraire de l'économie américaine au XIXe siècle explique les fluctuations du vote de gauche aux États-Unis; selon que les conditions économiques sont ou non favorables aux fermiers américains, ils votent selon le cas, à gauche ou à droite. L'aspect agraire du socialisme américain a été reconnu depuis longtemps. Dans son édition de 1848, le Webster américain donne au mot socialisme la définition suivante : "un état social dans lequel tous les citoyens possèdent la prospérité en commun; un nouveau terme pour agrairisme.” À partir des socialismes utopiques du début du XIXe siècle, le caractère agraire

5 Mentionnons que la première grande guerre, la Révolution russe de 1917, la crise des années 30, la deuxième grande guerre et la guerre froide de l'après-guerre expliquent aussi le peu de succès du socialisme après 1914. 
du socialisme américain s'est maintenu. Il semble qu'il n'y ait eu aucune difficulté chez plusieurs socialistes à concevoir qu'on puisse posséder la terre en commun, mais qu'ils concevaient moins facilement que la propriété des moyens de production pût, elle aussi, être commune. Quoi qu'il en soit, l'explication du caractère agraire du socialisme américain reste très partielle puisqu'elle ne rend aucunement compte qu'après 1914 la clientèle socialiste se disperse.

On est alors tenté d'examiner la vie et le système politiques des U.S.A. Si, en effet, les théories socialistes réclament, comme le dit Durkheim, "le rattachement plus ou moins complet de toutes les fonctions économiques ou certaines d'entre elles, même diffuses, aux organes directeurs et conscients de la société," ${ }^{6}$ comment de telles théories ont-elles chance de prendre pied dans un pays où les fondateurs et les légistes se sont donné explicitement pour tâche de minimiser le rôle de l'État. Les "Founding Fathers," comme l'a remarqué l'historien américain Commanger, "se sont arrangés pour qu'il soit difficile au gouvernement, non seulement d'empiéter sur les domaines qui lui sont interdits, mais même d'exercer les pouvoirs qu'il détient. Ils ont inventé un système destiné à mettre normalement le gouvernement américain dans l'impossibilité d'agir." 7 Le système politique américain est fait de freins et de contrepoids qui visent à restreindre le pouvoir de l'État et à lui faire jouer le rôle d'arbitre des intérêts individuels et à ne jamais prendre lui-même d'initiatives. Les partis politiques américains reflètent cet état de choses. "Étant donné, écrit Walter Adams, que les deux principaux partis sont des amalgames de groupes de pression et représentent l'un comme l'autre des accommodements entre intérêts opposés, ils doivent devenir des agglomérats sans idéologie ni programmes cohérents." Adams continue ainsi: "La modération, l'esprit de compromis et le manque de conscience de classe qui caractérisent la vie politique américaine s'expliquent par une autre série de facteurs ayant pour base les traditions d'individualisme, la croyance aux possibilités d'ascension sociale et l'acception d'une Weltanschaung [vision du monde] pragmatique qui ont toujours prévalu dans le pays." ${ }^{8}$ Il est bien évident qu'un parti socialiste qui possède une idéologie explicite et un programme cohérent ne s'insérera pas facilement dans la vie politique américaine. Il s'oppose trop carrément à l'idéologie individualiste américaine pour ne pas heurter de front trop de traditions respectables.

Si l'on se reporte à la thèse du sociologue américain Seymour Lipset qui veut faire des U.S.A. la première "nouvelle nation" à passer par le processus de décolonisation, on verra qu'on peut en tirer des conséquences différentes de

Durkheim, Émile, "Le Socialisme", Paris, 1928, p. 27.

Cité par Walter Adams, "Économie, Idéologie et Politique aux États-Unis", Diogène, no. 36, p. 59.

8 Durkheim, Émile, "Le Socialisme", Paris, 1928, p. 27. 
celles que Lipset propose. Le sociologue américain propose cette théorie pour faire du pied et de l'oeil aux autres jeunes nations d'Asie et d'Afrique qui, elles aussi, secouent aujourd'hui le joug du colonialisme comme les U.S.A. l'avait fait à la fin du XVIlle siècle; il leur propose le modèle américain, en leur disant que les U.S.A. sont leurs grands aînés dans cette lutte pour l'indépendance et la décolonisation. Se rend-on compte toutefois qu'il s'est écoulé presque deux siècles entre ces deux moments de la décolonisation et qu'un modèle qui a eu du succès en ce temps-là peut très bien ne pas correspondre aux nécessités économiques des années 1960. Particulièrement en ce qui touche l'individualisme et le collectivisme, il ne semble pas que Lipset ait tenu compte de tous les facteurs qui ont pu changer la situation dans un intervalle de deux siècles. Les révolutionnaires et les radicaux d'une période peuvent devenir les réactionnaires d'une autre période; les Américains, révolutionnaires de 1776 ne peuvent peut être plus servir de modèle aux révolutionnaires et aux radicaux d'aujourd'hui. Les mouvements radicaux du début de la période moderne - que les Américains ne feront que porter à leur terme s'opposent au "communalisme" du Moyen-Age et réclament l'individualisme économique; les radicaux du XVIe et du XVIIe siècles réclament l'émancipation de l'individu. La doctrine de l'individualisme américain se place dans cette lignée. "Aux yeux des premiers colons, dit Walter Adams, l'État servait surtout à créer des privilèges, à imposer des monopoles et à opprimer les libertés individuelles. En tant qu'Européens, beaucoup de ces hommes avaient souffert des persécutions religieuses organisées par des gouvernements intolérants; en tant que colons, ils devaient subir les discriminations et les restrictions imposées par le mercantilisme du régime; devenus citoyens libres, ils trouvèrent naturel d'admettre, avec Jefferson, que le meilleur gouvernement est celui qui gouverne le moins." 9 Personne ne songe à nier que les Américains n'avaient pas raison en 1776 mais les conditions ont bien changé depuis, et leur révolution et leur radicalisme ne peuvent servir d'exemple aux autres jeunes nations d'aujourd'hui. Depuis la dernière moitié du XIXe siècle, le radicalisme est accroché à l'autre axe du balancier démocratique: celui de l'égalité des hommes étant donné que les libertés civiles, religieuses et politiques ont été à peu près conquises. Cet individualisme et ce pragmatisme qui ont fait leur force et qu'on peut regarder "a posteriori" comme des conditions de leur développement économique doivent être considérés comme le résultat de certains processus historiques et non pas comme produits exportables.

Or ce pragmatisme de toute la société américaine se manifeste très tôt dans le mouvement travailliste américain. "Dès 1883, Adolf Strasser, l'un des chefs du mouvement répondait au comité du Congrès qui l'interrogeait sur les objectifs des syndicats : "Nous n'avons pas de but définitif. Nous allons notre chemin au jour le jour. Nous combattons seulement pour des objectifs immédiats, des objectifs que nous pourrons atteindre d'ici quelques années ... Nous

9 Ibidem, p. 63-64. 
sommes tous des hommes pratiques". Répondant à la même question en 1914, Samuel Gompers qui fut le premier président de l'American Federation of Labor, déclarait: "Le mouvement travaille selon une ligne de moindre résistance, il s'efforce d'obtenir les meilleurs résultats et d'améliorer la condition des travailleurs, hommes, femmes, enfants, au jour le jour, faisant de chaque jour un jour meilleur que celui qui l'a précédé..." ${ }^{10}$ Cette phrase manifeste non seulement le pragmatisme de l'Américain mais cette confiance en soi, la "selfreliance", dont parle Emerson. Expression Yankee d'une notion calviniste influencée par les idées éclairées du XVIIIe siècle, cet individualisme consistait simplement à croire que l'individu doit se charger personnellement de réaliser son propre bien-être moral, politique et économique. Pour expliquer donc le peu de succès qu'a eu le socialisme aux U.S.A., on peut invoquer la nature de la constitution américaine (freins et contrepoids) et le type des partis politiques (agglomérats d'intérêts privés); derrière eux, on retrouve l'individualisme et le pragmatisme de l'idéologie américaine.

\section{H. Idéologie américaine: Idéologie-substitut}

\section{$\underline{\text { Retour à la table des matières }}$}

Si l'on considère le socialisme comme une idéologie, c'est-à-dire l'expression conceptualisée des valeurs auxquelles sont attachés des individus et des groupes, on peut dire que cette idéologie s'est heurtée aux U.S.A. à une autre idéologie plus ou moins implicite : l'américanisme. Et cette idéologie, sousjacente à l'“American way of life" se propose plus ou moins comme une idéologie-substitut qui se donne comme la réalisation partielle de l'idéologie socialiste. En effet, le socialisme, né dans la vieille Europe, proposait d'édifier une société nouvelle, un homme nouveau, en transformant les anciennes structures sociales. N'est-ce pas ce que les U.S.A. sont en train de faire à partir de 1776 ? De Crècevoeur écrit en 1782 : "L'Américain est un homme nouveau qui agit d'après de nouveaux principes". Le socialisme du XIXe siècle ne fait que promettre de bâtir cette nouvelle société; l'Américain, lui, est en train de la réaliser. Le socialisme promet une société sans classe, l'Américain croit qu'il l'a réalisée : pas d'aristocratie, pas de privilèges; chacun peut devenir ce

10 Adams, Walter, Ibidem, p. 67. 
qu'il veut en faisant fructifier son talent. Pendant plus de trois cents ans de son histoire, les frontières de l'Amérique ne sont pas atteintes; pendant tout ce temps-là, des territoires sont découverts, colonisés, exploités, le rêve américain de richesses illimitées se poursuit. Pas de frontières étanches entre les différents groupes d'Américains. Un Américain peut être pauvre mais il pense que demain, il peut être riche; il peut être en chômage, mais il trouvera certainement de l'emploi demain.

Le socialisme - et cela à partir de Saint-Simon et non seulement de Marx pense qu'il peut accroître la production nationale; l'Amérique a déjà résolu ce problème-là et, pendant tout le XIXe siècle, sa croissance est foudroyante. Le socialisme promet la liberté pour tous. Depuis 1776, la constitution américaine a proclamé que tous les hommes sont égaux et libres. Y a-t-il un peuple au monde, pensent les Américains, plus libre qu'eux?

L'Américain est un homme qui se pense et se veut un homme nouveau dans un monde nouveau; il a définitivement rompu avec l'Europe. Les échos qu'il en a pendant le XIXe siècle et le début du XXe siècle ne le portent pas à accepter d'emblée ce qui lui arrive de ce continent. Les révolutions de 1848, la Commune de Paris en 1871, la révolution bolchévique de 1917 le confirment dans son idée que la vieille Europe n'a rien à apprendre à l'Amérique. Au contraire, l'Américain se voit, lui et sa civilisation, comme exceptionnels". Cet "exceptionnalisme" de l'Amérique que ses écrivains et plusieurs observateurs étrangers confirment, constitue une idéologie de remplacement au socialisme.

\section{Morale et politique}

$\underline{\text { Retour à la table des matières }}$

Daniel Bell, l'auteur de "The End of Ideology" a lui-même examiné quelques-unes des raisons que différents auteurs ont données pour expliquer la faillite du socialisme aux États-Unis. Pour Bell, la cause principale de cet échec vient de la tension qui existe entre la morale et la politique, tension que les socialistes américains n'ont pas su résoudre. En quoi consiste cette tension ? Elle vient de cette question fondamentale que leur posait le socialisme : accepter le capitalisme et essayer de le réformer de l'intérieur comme acceptèrent de le faire Gompers et les syndicalistes américains ou bien devenir les ennemis acharnés de cette société et prendre toutes les décisions qu'une 
telle position implique. Leur théorie leur commandait la seconde option, tandis que dans la pratique ils ont opté pour la première. Cette tension, ce dilemme n'existe pas pour les socialismes utopiques ni pour les communistes. Pour les socialistes utopiques, il ne s'agissait pas de réformer la société mais de vivre en marge de cette société et de sauver les quelques individus qui acceptaient de vivre dans les communautés que les socialistes fondent; ils acceptent de vivre dans le monde mais de n'être pas du monde.

Le socialisme dit scientifique qui s'accorde avec la variété utopique sur le diagnostic de la société capitaliste ne croit pas que la fondation de petites communautés va régler le problème du capitalisme. Il s'agit non pas de changer quelques individus mais de changer la structure globale de la société; c'est pourquoi il faut porter le débat sur la place publique, créer des partis politiques et ultimement prendre le pouvoir. C'est alors seulement que la société pourra être changée et que les réformes socialistes pourront prendre corps. Mais aussitôt se pose le problème que Bell considère comme le talon d'Achille du socialisme américain; la politique est-elle le moyen de réaliser un idéal ou le moyen d'obtenir des avantages dans les limites de la morale courante? Max Weber voit dans cette tension la différence entre l'éthique de la responsabilité et de la conviction. C'est le problème classique des fins et des moyens. Le communiste ne se pose pas ce problème; il agit d'après l'éthique de conviction : il rejette la société capitaliste et ne se croit pas lié par les règles de cette société. Seul importe pour lui le renversement de la société bourgeoise, peu importe les moyens qu'il lui faudra employer.

Le socialiste, lui, joue toujours sur une corde tendue; parce qu'il est démocratique il ne peut pas ne pas être attentif aux moyens qu'il emploie; parce qu'il est dans le monde et de ce monde, il doit essayer de réaliser un mouvement dialectique entre l'éthique de responsabilité et de conviction; il ne doit pas perdre de vue ses convictions politiques et idéologiques d'une part et il doit se conformer à la volonté du peuple. Il est une autre tension qu'il doit aussi subir et qui le guette et, cette fois-ci, le communiste n'en est pas exempt: d'une part, il croit que le sens de l'histoire va vers le socialisme; d'autre part, il fonde un parti politique, fait de l'action politique et veut ainsi pousser à la roue de l'histoire. Dans quelle mesure sera-t-il tenté de se fier au déterminisme de l'histoire qui, croit-il, va dans son sens et ne pas contester la politique capitaliste au jour le jour ? Ces deux dilemmes ont toujours assailli les socialistes et ne sont donc pas spécifiques au socialisme américain. jusqu'ici on ne voit pas pourquoi Daniel Bell croit y déceler la raison majeure de l'insuccès du socialisme aux U.S.A.

Il semble bien qu'il faut faire mention d'un autre élément spécifiquement américain, c'est la nature et l'origine religieuse du socialisme américain. Le socialisme utopique religieux a autant et sinon plus réussi aux U.S.A. qu'en Europe alors que le socialisme scientifique, lui, n'a jamais eu là l'importance 
qu'il a eue en Europe. Selon Bell, même ceux qui se réclamaient du socialisme scientifique aux U.S.A. n'avaient pas été amenés à contester le capitalisme après une analyse froide et rationnelle de la société mais c'était l'indignation devant la pauvreté et la promesse évangélique d'un monde meilleur (lui les avait attirés vers le socialisme. (On n'a qu'à se reporter aux socialistes anglocanadiens (néo-démocrates) pour constater comment des ministres protestants deviennent des réformateurs politiques. Un Knowles et un Douglas, en sont de bons exemples.) Dans les pays européens, socialisme et laïcisme et, au XIXe siècle, anticléricalisme et socialisme faisaient bon ménage. Selon Bell encore, l'origine religieuse du socialisme américain se serait révélée par le fractionnement du mouvement en différentes tendances et en de nombreuses sectes tout comme le protestantisme a lui-même proliféré en sectes religieuses. Le conflit entre la morale et la politique est accentué par la disparité entre l'idéal religieux qui n'est pas de ce monde et l'action qui, elle, est de ce monde.

\section{En guise de conclusion}

\section{$\underline{\text { Retour à la table des matières }}$}

Nous ne chercherons pas à discerner la raison ou le faisceau de raisons qui rendraient mieux compte de la faillite électorale du socialisme aux États-Unis. Disons simplement que ce pays a bénéficié de conditions économiques particulièrement favorables et que le capitalisme concurrentiel d'abord et monopolistique de nos jours a pu s'y développer librement. La "première nouvelle nation", comme l'appelle Lipset, a bouclé la boucle du libéralisme et du capitalisme. Dans cette société, dans ce continent qui possédait tous les atouts pour pousser au bout l'expérience de la libre entreprise et qui a raffiné l'art de l'abondance et du gaspillage, le socialisme n'a pu se constituer une clientèle électorale. Doit-on en conclure pour cela qu'il n'a aucun avenir aux ÉtatsUnis ? Et que les autres sociétés qui veulent atteindre le même niveau de vie que les Américains doivent nécessairement employer les mêmes techniques économiques et sociales et s'inspirer de la même idéologie ? Même ceux des Américains qui tiennent fermement au maintien des structures capitalistes aux États-Unis commencent à admettre que le socialisme se présente aux pays économiquement sous-développés comme une technique de rattrapage du capitalisme. Un récent numéro du "Scientific American" sur "la technologie et le développement économique" concluait qu'il existe beaucoup de raisons 
objectives aussi bien que des raisons idéologiques pour lesquelles le rôle de l'État devrait être primordial dans la planification et le développement économique. C'est d'ailleurs la voie qu'empruntent aujourd'hui la plupart des pays sous-développés dans plusieurs parties du monde. Si un certain nombre d'analystes néo-libéraux concèdent que le socialisme leur apparaît comme valable pour hâter le décollage économique des pays en voie de développement, ils affirment ou laissent entendre que la société néo-libérale va résoudre les contradictions du capitalisme et se passer elle-même du socialisme. Ce n'est pas le moment de dire, dans cet article, que la critique de la société néolibérale, qu'elle soit américaine ou européenne, est commencée depuis longtemps; le moins qu'on puisse affirmer, c'est qu'il est loin d'être sûr que la société néo-libérale, pas plus que la société libérale, pourra instaurer une véritable démocratie économique et sociale. Comme le dit excellemment André Philip 11 "l'entreprise n'exprime plus un droit de propriété mais une fonction sociale". Même le temps du socialisme distributiviste, du "Welfare State" est passé; on ne peut plus aujourd'hui "laisser au capitalisme la responsabilité de l'organisation de la production, en prélevant sur ses profits de quoi améliorer le sort des masses. C'est cette organisation elle-même qui aujourd'hui est remise en question". Ce ne seront probablement pas, pense George Lichtheim ${ }^{12}$, les pays capitalistes les plus puissants économiquement qui deviendront socialistes dans l'avenir immédiat; ce seront plutôt des sociétés de moyenne importance qui, pour sortir de leurs contradictions socio-économiques, pourront se diriger plus librement vers le socialisme, sans mettre en danger l'équilibre précaire des forces politiques dans le monde.

Que l'insuccès électoral du socialisme aux États-Unis tienne plus au cas américain lui-même qu'au continent ou au type de société, nous est fourni par le succès relativement plus élevé que le socialisme a connu au Canada. Un gouvernement de type socialisme distributiviste a même conquis et gardé le pouvoir en Saskatchewan depuis près de vingt ans. Ce qui veut dire que le cas américain mis à part, la très grande majorité des pays occidentaux ont accordé des succès électoraux au socialisme, sinon le pouvoir dans bien des cas.

Qu'en est-il du Québec? Du Québec qui sort à peine de sa société traditionnelle, qui vient seulement de se découvrir une vocation de société industrielle et qui ne fait qu'ébaucher le projet de s'occuper lui-même de sa croissance économique et de vouloir se rendre maître de son destin économique et politique ? N'est-il pas bien téméraire d'espérer que sur un continent où le colosse américain a toujours rejeté le socialisme comme idéologie et comme pratique socio-économique, dans une confédération où le socialisme n'a connu que des succès mitigés, le socialisme s'implante dans un État, celui

Philip, André : "Le sort de la démocratie française" Preuves, sept. 1963, p. 8.

Lichtheim, George : "The Future of Socialism" Partisan Review, Spring 1963, p. 98. 
du Québec, qui a toujours passé pour la région la plus arriérée du continent nord-américain?

D'abord, le cas du Québec n'est en rien comparable à celui des États-Unis. Région qui, au Canada, selon Rostow ${ }^{13}$ se compare au sud des États-Unis par son retard économique, elle est composée en grande majorité par des Canadiens français qui sont et se considèrent de culture différente des autres Canadiens. Dominé économiquement par des intérêts étrangers, minorité à l'intérieur du Canada, ce peuple réclame aujourd'hui son autonomie politique pour pouvoir réaliser sa libération économique. Il n'est pas sûr qu'il ne devra pas emprunter la même technique de rattrapage économique que les autres pays, hier colonisés, emploient aujourd'hui pour bâtir leur économie. Si l'évolution sociale suit la même stratégie que l'évolution biologique et qu'elle s'accomplit par sauts plutôt que par le progrès constant des organismes les plus perfecdonnés, le Québec peut espérer faire un bond plus vite vers la démocratie économique et sociale que les sociétés oppulentes qui, elles, ont partie liée avec le vieil ordre des choses. C'est ce que Trotsky appelait "le privilège du retard historique". Peut-être que Toynbee avait la même idée quand il écrivait: "Si c'est un avenir heureux dans l'ensemble qui attend l'humanité, alors je prédirais volontiers qu'il y a de l'avenir, dans le vieux monde pour les Chinois, et dans l'île d'Amérique du Nord pour les Canadiens. ${ }^{14}$ Quel que soit l'avenir de l'humanité en Amérique du Nord, je suis pour ainsi dire sûr que ces Canadiens de langue française, en tout état de cause, seront encore présents au dénouement de l'aventure". 15

\section{Marcel RIOUX}

Fin du texte

Rostow, W. W. : "The Stages of Economic Growth" Cambridge University Press, N.Y. 1960, p. 67.

14 En français dans le texte.

15 Toynbee, Arnold J.: "La civilisation à l'épreuve", Paris, 1951, p. 176-177. 